\title{
Automated Human Recognition by Gait using Neural Network
}

\author{
Jang-Hee Yoo \\ Information Security \\ Research Division, ETRI \\ S. Korea \\ Ki-Young Moon \\ Information Security \\ Research Division, ETRI \\ S. Korea
}

\author{
Doosung Hwang \\ Department of Computer Science, \\ Dankook University \\ S. Korea \\ Mark S. Nixon \\ School of Electronics and Computer \\ Science, University of Southampton \\ United Kingdom
}

\begin{abstract}
We describe a new method for recognizing humans by their gait using back-propagation neural network. Here, the gait motion is described as rhythmic and periodic motion, and a $2 D$ stick figure is extracted from gait silhouette by motion information with topological analysis guided by anatomical knowledge. A sequential set of $2 D$ stick figures is used to represent the gait signature that is primitive data for the feature extraction based on motion parameters. Then, a back-propagation neural network algorithm is used to recognize humans by their gait patterns. In experiments, higher gait recognition performances have been achieved.
\end{abstract}

Key Words- Gait, Gait Biometrics, Human Motion

\section{Introduction}

The study of human gait has been increased extensive interests in various fields such as clinical analysis, computer animation, athletic performance analysis, visual surveillance, robotics, and biometrics [6]. Moreover, human motion analysis has many challenging issues, because the highly flexible structure and self-occlusion of the human body mandates complicated processes for the segmentation and analysis of motion [13]. As the human gait is known to be one of the most universal and complex of all human activities, it has been studied in medical science [9][15][16], biomechanics [19][20], and psychology [11] for decades. In computer vision, automated person identification by gait has been investigated [12].

The human gait is usually described by kinetic or kinematic characteristics [1], and the pattern of rotation angles around the joints has been modeled as a pendulum [19][20], the motion of which is characterized by simple harmonic motion. In addition, each person appears to have his or her own characteristic of gait pattern. There is much evidence from psychophysical experiments [11] and gait analysis [9][15] that gait patterns have characteristics of uniqueness for each individual. As a biometric, human gait may be defined as a means of identifying individuals by the way they walk [12]. Using gait has many advantages over other biometrics such as fingerprints, most notably that it is non-invasive and available at low resolution.

In this paper, we propose a new method for recognizing humans by their gait using back-propagation neural network. To extract gait motion, the human body and its silhouette is extracted from the image sequences. Then, a 2D stick figure is extracted from body silhouette by determining the body points. Also, trajectory-based kinematic features are calculated from gait sequences for analyzing the gait motion. Then, an enhanced backpropagation neural network algorithm is employed to identify the gait features. As such, the new system aims to derive measures of biometrical significance, in part demonstrated by recognition capability.

\section{Describing Human Gait Motion}

Human gait has common patterns of movements and describes a rhythmic and periodic motion by which the body moves step by step in the required direction. A period of the gait cycle exists between the successive heelstrikes, and the gait motion in space and time satisfies spatial and temporal symmetry. To extract the gait motion, body angles of each segment are extracted from gait silhouette images. A simplified 2D stick figure with six joint angles [21] is used to represent the human body structure for recovering and interpreting the human movement.

\subsection{Human Gait as a Periodic Model}

Human gait is described by a process of locomotion in which the moving body is supported by first one leg and 
then other. As the moving body passes over the supporting leg, the other leg is swung forward in preparation for its next stance phase [9][16]. The time interval between two successive occurrences of one of the repetitive events of gait motion is defined as the gait cycle [15]. Each gait cycle is separated into two distinct periods: the stance and the swing phase. In general, the stance and swing phases have been divided into eight sub-phases: initial contact $(I C)$, loading response $(l r)$, mid-stance $(M S)$, terminal stance $(t s)$, pre and initial swing $(p w)$, mid-swing $(M W)$, and terminal swing $(t w)$ [9]. Each of the eight gait phases has a functional objective and a different length time interval in gait cycle.

Also, human gait motion has many modes, among which walking, running and standing are the three most often seen in daily life. These modes are the states of action in human motion, and a human can change between these states. By biomechanics definitions, standing, walking and running are distinguished firstly by the stride duration, stride length, velocities and the range of motion made by the limbs. That is, the speed of the gait motion is the most important feature to distinguish among these three states. A second difference concerns the existence of periods of double support or double float, and this is determined by the duration of the stance phase. In general, normal walking is translated to double support $(D S / I C)$ states through the single support $(s s)$. The relationship of the states is naturally represented as a finite state machine as shown in Figure 1.

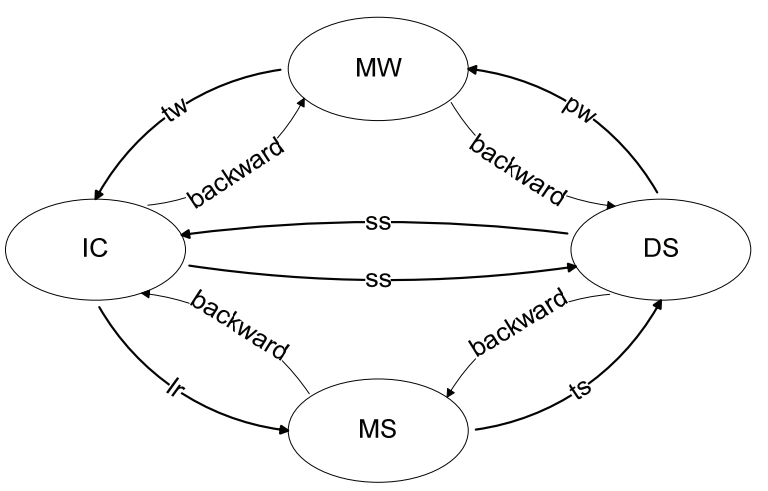

Figure 1. Transition Diagram for Gait Motion

However, the changes in joint motion that occur during each phase are described by the joint ranges of motion at each segment of gait. Statistical analyses of the gait relationships typically use continuous curves of the time series data measured over the gait cycle. Medical research [15] has shown that the pattern of gait motion is approximately sinusoidal in nature. Therefore, trigonometric functions are naturally suited to estimating a gait curve from time series data. Consequently, an assumed functional relationship between periodic gait motion and time can be modeled by interpolation of trigonometric polynomials. Also, further gait motion can be predicted by the periodicity of this interpolation model. An $n^{\text {th }}$-order trigonometric polynomial interpolant function is

$$
\begin{aligned}
y_{n}(x)= & a_{0}+a_{n} \cos (2 \pi n x) \\
& +\sum_{k=1}^{n-1}\left[a_{k} \cos (2 \pi k x)+b_{k} \sin (2 \pi k x)\right]
\end{aligned}
$$

where the $a_{0}, a_{n}, a_{k}$ and $b_{k}$ are unknown curve-specific coefficients. As $n \rightarrow \infty, y_{n}(x)$ tends to the Fourier series.

\subsection{Human Gait Database}

Early gait databases reported in the literature were collected under very limited conditions and consisted of a relatively small number of subjects. To overcome this, larger databases have been developed by DARPA Human ID projects [17]. The SOTON database [18] developed by the ISIS Research Group is one of the largest databases in the DARPA project. The SOTON database comprises two forms of indoor data (under controlled lighting with special background) and one outdoors (without lighting or background control). The first form of indoor data is a subject walking on a treadmill, and the second form is subject walking alone a specially designed continuous track.

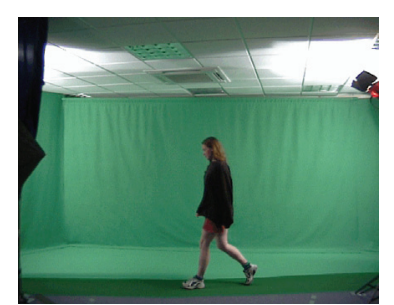

(a) Indoor Data

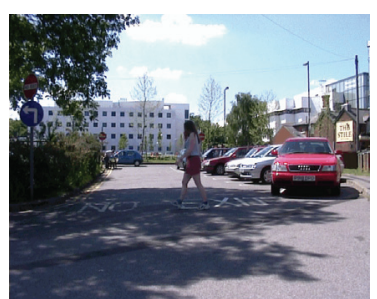

(b) Outdoor Data
Figure 2. Images from SOTON Database

Sample images from the database are shown in Figure 2. As can be seen in Figure 2(a), chroma-key laboratory was constructed to allow controlled lighting conditions for the indoor treadmill and track data. An image sequence contains only a single subject walking at normal speed and was acquired at 25 frames / second with $720 \times 576$ colour pixels from a DV camera. Also, the subject was filmed walking in both directions, and the database contains more than 100 subjects. Accordingly, a marker-less analysis system can contribute to the debate concerning the use of a treadmill versus normal walking, for a large number of subjects.

In addition, human body extraction from the image sequences is easily achieved through background subtraction. After that, the histogram projection profiles are ana- 
lysed to estimate the position of a human body, and the body region is verified by prior knowledge such as size and shape. Thresholding and morphology is then used to extract the contour of a detected human body. Here, a thresholding method based on similarity measures between the background and the object image is used [21]. Finally, the body contour is extracted by subtraction followed by dilation and erosion. Thresholding is a simple image segmentation method, and erosion will remove the outer layer of pixels from an object.

\subsection{Extracting Gait Signature and Feature}

To extract body points in a gait silhouette image, a skeleton data with body segment properties is used [5]. For a body height $H$, an initial estimate of the vertical position of the neck, shoulder, waist, pelvis, knee and ankle was set by study of anatomical data to be $0.870 \mathrm{H}$, $0.818 H, 0.530 H, 0.480 H, 0.285 H$, and $0.039 H$, respectively [5][21]. The gait skeleton can be simply calculated by two border points of each body part $p$ with a range constraint. The angles $\theta_{p}$ of body part $p$ from skeleton data can be approximated by using the slope of the lines in linear regression equation. The body angles around three double support phases are clearly extracted, but the angles around single support phases are not as accurate as those estimated for the double supports. Therefore, a motion tracking method between double supports is used to extract body points at the lower limbs as well.

The nine coordinates (body points) are extracted from the gait silhouette data, and the $2 \mathrm{D}$ stick figures are obtained by connecting the nine coordinates. Figure 3 shows the extracted body silhouettes and their stick figures during one gait cycle. The gait signature can be defined as a sequence of the stick figures obtained from gait silhouette data during one gait cycle. The stick figure model is the most effective and well-defined representation method for kinematic gait analysis. Moreover, the stick figure is closely related to a joint representation, and the motion of the joints provides a key to motion estimation and recognition of the whole figure.

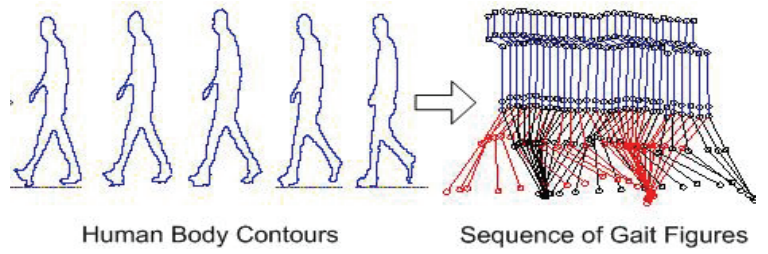

Figure 3. Human Gait Signature

On the other hand, the mean and variation of the gait angles for single sequence of each subject are extracted as features. The variation of the gait angles can be obtained by standard derivation during one gait cycle. Trajectories of gait figure contain kinematic characteristics on human movement. The kinematic characteristics include linear and angular position, their displacements and the time derivatives, notably the linear and angular velocities and accelerations. As a feature, the gait velocity $v_{t}$ at frame $t$ is calculated by

$$
v_{t}=\left(x_{t}-x_{t-1}\right) / \Delta t .
$$

Also, the angular velocities $\omega_{t}$ at frame $t$, given an interframe time $\Delta t(1 / 25 \mathrm{sec})$, are

$$
\omega_{t}=\left(\theta_{t}-\theta_{t-1}\right) / \Delta t .
$$

The trajectories are basically vector-valued function at each frame $t$ of an image sequence, and they are periodic as in gait motion.

\section{Automated Human Recognition by Gait}

Automated person identification is an important task in many security systems such as video surveillance and access control. It is well-known that biometrics are a powerful tool for reliable automated person identification [6][12]. Automatic gait recognition is one of the newest of the emergent biometrics and has many advantages over other biometrics. The most notable advantage is that it does not require contact with the subjects nor does it require the subject to be near a camera. Various approaches [12] for the classification and recognition of human gait have been studied, but human gait identification is still a difficult task. Here, the gait feature vectors extracted from the gait signatures of the SOTON database are used for designing the neural network architecture.

\subsection{Human Gait Identification System}

In general, a biometric system operates either as a verification or as an identification system. A verification system authenticates a person's identity by comparing the input biometric characteristic with a person's own biometric data pre-stored in the system, so the system either rejects or accepts the submitted claim for authentication. An identification system recognizes an individual by searching the template database for a match [10]. However, a typical human gait identification system can be divided into training and recognition modules. The training module is responsible for making a trained database to identify a person. During the training phase, the gait motion is captured by a video camera for acquiring a digital representation of the characteristic. A feature extractor processes this representation to generate a more compact and expressive representation such as gait feature vector. The feature vectors for each person are then trained by a pattern recognition algorithm, and the trained results will be stored in a gait identification system's database. 


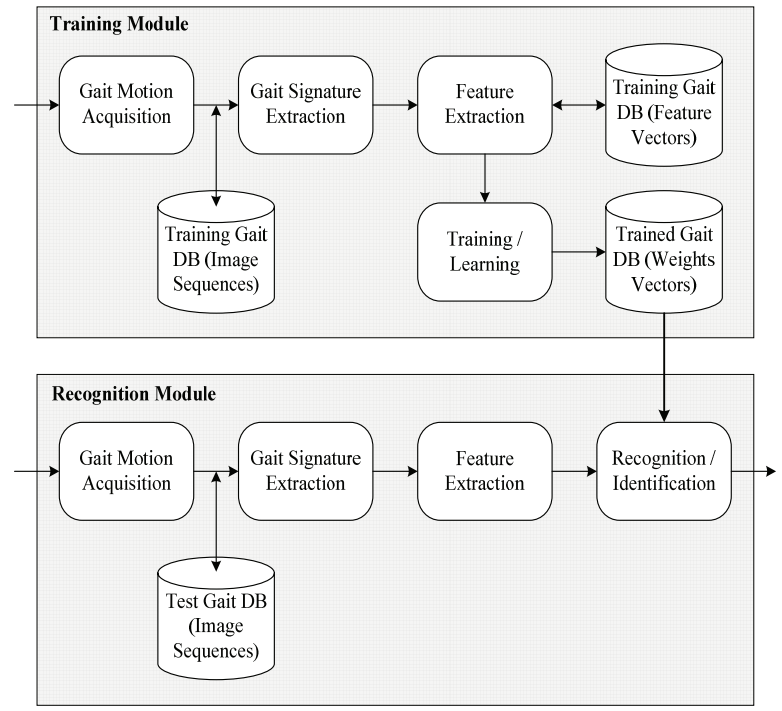

Figure 4. Block Diagram of Gait Identification System

In addition, the recognition module is responsible for identifying the person. During the recognition phase, the video camera captures the gait motion of the person to be identified, and it converts into the same sort of feature vector as in training. After that, the feature vector will be submitted to the recognizer, which automatically computes it against the trained database to determine the identity of the individual. Figure 4 shows the block diagram of our gait identification system. To train and recognize the gait, a multi-layered neural network is used. The architecture of a typical biometric system also consists of same components.

\subsection{Neural Network for Gait Identification}

The pattern classifier (or recognizer) is one of the most important components of the gait identification system. Many approaches to analyze and recognize gait have been used [3][4][12]: $k$-nearest neighbours, fuzzy clustering, principal component analysis (PCA), canonical analysis, neural networks, fractal dynamics, and wavelet methods. Recently, Chau [3][4] has reviewed these approaches for analyzing and classifying gait data. He notes that neural network methods facilitate gait analysis because of their highly flexible, inductive, non-linear modelling ability, unlike any other approaches. The non-linear property of multi-layered neural networks is useful for analysis of complicated gait variable relationships which have traditionally been difficult to model analytically. Hence, an enhanced back-propagation algorithm for training multi-layered neural network, based on selective retraining and a dynamic adaptation of learning rate and momentum, is employed to recognize the gait.

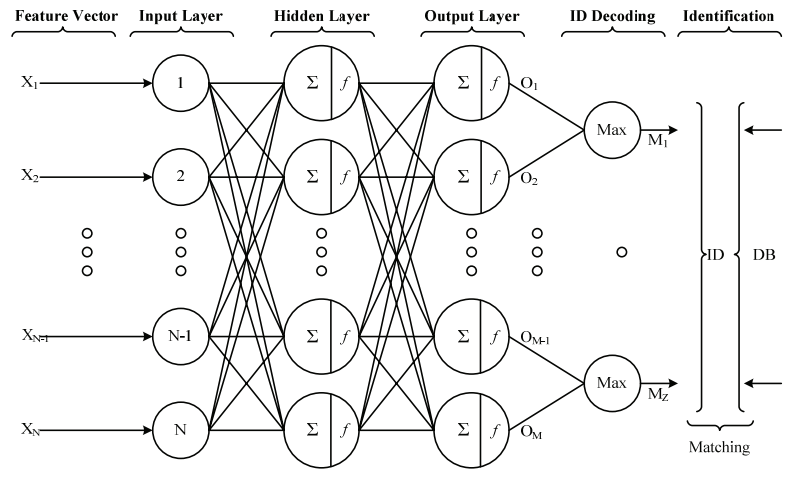

Figure 5. A Two-Layer Neural Network

An automated pattern recognition system minimally contains an input subsystem that accepts sample pattern vectors and a decision-maker subsystem that decides the classes to which an input pattern vector belongs [14]. If it also classifies, then it has a training phase in which it learns a set of classes of the population from a sample of pattern vectors, namely, it partitions the population into the subpopulations that are classes. As described in the previous section, a multi-layered feed-forward neural network is employed here to train and recognize the human gait. Figure 5 shows the network architecture used within this study. In the figure, $\mathbf{X}$ is the input feature vector with $N$ elements, and $\mathbf{O}$ is the output vector with $M$ elements. The neural network has one hidden layer of sigmoid nodes followed by an output layer of linear nodes. To train the network, the enhanced back-propagation algorithm is used. Also, the nodes of output layer are divided into two groups, and information about a maximum output node of each group is used to decode the output to an identification code of gait.

\section{Experimental Results}

In the experiments, 90 different subjects with seven gait signatures of each subject, a total of 630 gait signatures, are used. The subjects are categorized into three different groups according to the quality levels of the preprocessed body contour data, and each group contains 30 different subjects. To apply the neural network, four gait signatures of each of the 30 subjects for each group are used to generate the 150 training feature vectors, and the arithmetic means of the other three gait signatures of each of the 30 subjects for each group are used to generate the 30 test feature vectors. Also, the 10 gait features for each subject are used as input data, and the numbers of hidden nodes and output nodes are set to 28 and 13 respectively. To decode the output to identification code, the first digit of the code is determined by a maximum output node among the first three output nodes, and the second digit of 
the code is determined by the other output nodes. To recognize humans by their gait, the neural networks are trained until recognition on the training data reached 100 percent, thus the classification rates for each group of the training sets were $100 \%$.

Table 1. Experimental Result

\begin{tabular}{|c|c|c|c|c|}
\hline \multirow{2}{*}{$\begin{array}{l}\text { Body Contour } \\
\text { Quality } \\
\text { (\# of Subjects) }\end{array}$} & \multicolumn{2}{|c|}{$\begin{array}{l}\text { \# of Feature } \\
\text { Vectors }\end{array}$} & \multicolumn{2}{|c|}{ Recognition Rate } \\
\hline & $\begin{array}{l}\text { Train- } \\
\text { ing }\end{array}$ & Test & $\begin{array}{l}\text { Tra } \\
\text { Pha }\end{array}$ & $\begin{array}{l}\text { Recog. } \\
\text { Phase }\end{array}$ \\
\hline Class A - Good & 150 & 30 & $100.0 \%$ & $90.0 \%$ \\
\hline Class B - Fair & 150 & 30 & $100.0 \%$ & $83.3 \%$ \\
\hline Class C - Bad & 150 & 30 & $100.0 \%$ & $83.3 \%$ \\
\hline
\end{tabular}

Table 1 shows the recognition results for each group of the different quality levels. As can be seen in the table, the better the quality of results of the pre-processing stage, the better the performance of the recognition rate, and the neural network approach achieved a recognition rate of up to $90 \%$ for 30 subjects. Although the recognition rate does not reach $100 \%$, this study does suggest the possibility of this gait biometric. Naturally we seek to improve the recognition rate in future. Notwithstanding this, the marker-less gait recognition can clearly handle a small number (30) of subjects successfully as shown in other studies, often for an even smaller population. By this, these approaches show that people are unique by their walking pattern, according with earlier biomechanical suggestions, and buttressing other similar results.

\section{Conclusions}

We have described an automated gait recognition system using back-propagation neural network algorithm. Gait is the most common human motion, and each person appears to have his or her own characteristic gait pattern. To recognize the human gait, a total of 27 parameters are considered as gait features. By measuring a class separability of the given feature, only 10 important features for classifying the gait are selected from these feature sets. Then, the enhanced back-propagation neural network algorithm is applied to the SOTON database, and recognition rate of $90 \%$ for 30 subjects is accomplished. The results achieved give promising performance and higher recognition rates than those of an earlier gait recognition approach.

\section{References}

[1] J. K. Aggarwal, Q. Cai, W. Liao, and B. Sabata, "Nonrigid Motion Analysis: Articulated and Elastic Mo- tion", Computer Vision and Image Understanding, 70(2), pp.142-156, May 1998.

[2] J. G. Barton and A. Lees, "An Application of Neural Networks for Distinguishing Gait Patterns on the Basis of Hip-Knee Joint Angle Diagram", Gait and Posture, 5(1), pp.28-33, 1997.

[3] T. Chau, "A Review of Analytical Techniques for Gait Data. Part 1: Fuzzy, Statistical and Fractal Methods", Gait and Posture, 13(1), pp.49-66, 2001.

[4] T. Chau, "A Review of Analytical Techniques for Gait Data. Part 2: Neural Network and Wavelet Methods", Gait and Posture, 13(2), pp.102-120, 2001.

[5] W. T. Dempster and G. R. L. Gaughran, "Properties of Body Segments Based on Size and Weight", American Journal of Anatomy, 120, pp.33-54, 1967.

[6] D. M. Gavrila, "The Visual Analysis of Human Movement: A Survey", Computer Vision and Image Understanding, 73(1), pp.82-98, January 1999.

[7] A. Goswami, "A New Gait Parameterization Technique by Means of Cyclogram Moments: Application to Human Slop Walking”, Gait and Posture, 8(1), pp.15-36, 1998.

[8] M. K. Hu, "Visual Pattern Recognition by Moment Invariants", IRE Transactions on Information Theory, IT-8(8):179-187, February 1962.

[9] V. T. Inman, H. J. Ralston, and F. Todd, Human Walking, Williams \& Wilkins, 1981.

[10] A. K. Jain, R. M. Bolle, and S. Pankanti, "Introduction to Biometrics", in BIOMETRICS - Personal Identification in Networked Society, A. K. Jain, R. M. Bolle, and S. Pankanti, Eds., pp.1-41, Kluwer Academic Publishers, January 1999.

[11] G. Johansson, Visual Perception of Biological Motion and a Model for Its Analysis, Perception and Psychophysics, 14(2), pp.201-211, 1973.

[12] M. S. Nixon, T. Tan, and R. Chellappa, Human Identification based on Gait, Springer, 2006.

[13] M. K. Leung and Y. H. Yang, "First Sight: A Human Body Outline Labeling System", IEEE Transactions on Pattern Analysis and Machine Intelligence, 17(4), pp.359-377, April 1995.

[14] C. G. Looney, Pattern Recognition using Neural Networks: Theory and Algorithms for Engineers and Scientists, Oxford University Press, 1997.

[15] M. P. Murray, A. B. Drought, and R. C. Kory, "Walking Patterns of Normal Men", Journal of Bone and Joint Surgery, 46A(2), pp.335-360, 1964.

[16] J. Perry, Gait Analysis: Normal and Pathological Function, Slack, 1992.

[17] P. J. Phillips. Human Identification Technical Challenges. in Proceedings of the IEEE 2002 International 
Conference on Image Processing, pp.49-52, Rochester, USA, September 2002.

[18] J. D. Shutler, M. G. Grant, M. S. Nixon, and J. N. Cater. On a Large Sequence-based Human Gait Database. in Proceedings of Recent Advances in Soft Computing, pp.66-71, Nottingham, UK, December 2002.

[19] G. Taga, "A Model of the Neuro-Musculo-Skeletal System for Human Locomotion”, Biological Cybernetics, 73(2), pp.97-111, 1995.

[20] D. A. Winter, The Biomechanics and Motor Control of Human Movement, $2^{\text {nd }}$ Eds., John Wiley \& Sons, 1990.

[21] J. H. Yoo and M. S. Nixon, "Markerless Human Gait Analysis via Image Sequences", Proceedings of the ISB XIX ${ }^{\text {th }}$ Congress, Dunedin, New Zealand, July 2003. 